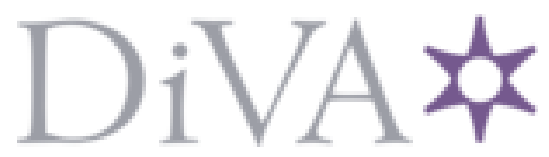

http://www.diva-portal.org

This is the published version of a paper presented at The 2013 IEEE International Conference on Mechatronics and Automation (ICMA 2013).

Citation for the original published paper:

Dandan, K., Ananiev, A., Ivan, K. (2013)

SIRO: the silos surface cleaning robot concept.

In: (pp. 657-661). IEEE conference proceedings

N.B. When citing this work, cite the original published paper.

Permanent link to this version:

http://urn.kb.se/resolve?urn=urn:nbn:se:oru:diva-30689 


\title{
SIRO: the silos surface cleaning robot concept
}

\author{
K. Dandan*, A. Ananiev, I. Kalaykov
}

\begin{abstract}
A concept of a suspended robot for surface cleaning in silos is presented in this paper. The main requirements and limitations resulting from the specific operational conditions are discussed. Due to the large dimension of the silo as a confined space, specific kinematics of the robot manipulator is proposed. The major problems in its design are highlighted and an approach to resolve them is proposed. The suggested concept is a reasonable compromise between the basic contradicting factors in the design: small entrance and large surface of the confined space, suspension and stabilization of the robot.
\end{abstract}

\section{INTRODUCTION}

Cleaning a silo is a tedious work and very dangerous job for humans due to many factors such as: unsafe oxygen level, engulfment, biological, mechanical, electrical, and atmospheric hazards [1]. The requirements of the EU norms related to hygiene and food quality imply that silos should be cleaned more frequently and obligatory after a silo is totally emptied. Therefore, there is an increased societal need of silo cleaning and a natural necessity of replacing humans by robot manipulators in executing this risky and dangerous job.

In a previous study a detailed survey of existing technologies and solutions for cleaning in large confined spaces shows that to clean the entire volume of the silo, solutions as hydraulic and pneumatic whips and augers, cardox tubes [2], acoustic cleaner [3] are very effective to remove the build up materials. These tools operate and interact directly with the material that is inside the confined space in order to release the jams and allow emptying the space. The used techniques are characterized by low precision, rough design, careless about contamination, high tolerance against remaining material staying on the walls, therefore they don't fit the goal of achieving fine cleaned interior surface.

Cleaning and sanitation of the interior surface requires another approach, namely the cleaning tool(s) have to interact with the surface only, but not to operate inside the big volume of the confined space. When talking about food silos, special focus is not only at taking out the material from the volume, but at removing all small pieces and particles from the silo surface. The implication is that a solution based on robot manipulator has to interact with the silo surface.

Due to the typically large dimension of a silo, the amount of possible technical arrangements is not big. Most of them are related to the above mentioned solutions, and three groups could be summarized:

- Suspended manipulators: the cleaning apparatus is suspended inside the confined space with steel cables,

Authors are is with School of Science and Technology, Örebro University, 70182 Örebro, Sweden.

* Corresponding author, kinan.dandan at oru.se chains or beams [4], [5]. Those robots can be applied effectively within a small confined space, but it is very complicated to implement them in large silo, because very long beams cannot be installed inside the silo, which is the main meaning of their concept.

- Rigid and flexible arm manipulators: used for removing the nuclear waste from the nuclear plants [6], for handling tasks [7], [8]. This solution is used in maximum height up to 10 meters and it needs wide space outside the confined space to install the mechanism, which in most cases is not available. In addition, the payload is too small for long arms and no additional forces can be tolerated.

- Climbing robots: development and applications grow up rapidly in the last decades specially in dangerous and hazardous environment. The robots stay and operate closest to the surface, and have a significant payloads. They use grippers [9] to grab firmly structural element available on the surface, or various types of adhesion, such as magnetic adhesion [10] used to interact with ferromagnetic surface or such elements on the surface, or vacuum adhesion [11] for non rough and relatively clean surfaces. Large silo are normally made of concrete and there is no ferromagnetic surface or any other elements to apply magnetic adhesion or grippers. Vacuum adhesion is easy to implement, but normally the surface of food silos is covered by a layer of particles and seeds, which strongly deteriorates the adhesion conditions.

Our analysis summarizes the following major conclusions for designing a surface cleaning robot:

- The majority of the existing cleaning equipment is constructed for cleaning the entire silo volume, but not for cleaning and sanitation of the interior surface.

- Most robots are used for inspection and maintenance tasks where the payload is too small.

- Most existing cleaning robots are used in small and metal confined space.

The field of cleaning, sanitation and assessment of confined spaces and interior surfaces formulates a number of specific requirements, which are not met by the existing commercial products. The idea of combining several particular solutions, elements of them or integrating several tools into a system, which could resemble a robot-like mechanism definitely does not work. Therefore, a specialized robot has to be developed for the silo cleaning and sanitation branch.

In this paper we present the design concept of interior surface cleaning robot for silos - the SIRO robot (SIlo RObot). Some of its functionalities, e.g. adaptable form 
varying from compact (to enter the silo) to expanded (when operating), mobility to move within the entire silo height, and stabilization at given operating point, are included and implemented in the concept. Some less important elements like the cleaning tools arrangement, sensing/perception and control are subject of another paper. Section II summarizes design requirements and Section III presents the SIRO concept. The basic locomotion of SIRO is discussed in Section IV. Concluding remarks are given in the last section.

\section{DESIGN REQUIREMENTS}

A typical food silo has a cylindrical shape with a $20-$ $30 m$ height and $4-8 m$ diameter, with cement surface. The bulk material is fed at the top and taken out at the bottom. Pesticides, insecticides and other toxic materials for cleaning are forbidden, since food products are stored in the silos. Dry cleaning with the pressurized air (air jets) is the preferable and recommended cleaning method, because wet cleaning of silo with concrete walls will create difficult to dry moisture. There is at least one circular (diameter $80 \mathrm{~cm}$ ) or rectangular $(80 \times 80 \mathrm{~cm})$ inspections hole placed on the silo roof, usually not placed at the central vertical axis of the silo. Today normally humans called "silo-divers" are lowered through this small hole. In addition cleaning instrumentation like air jets connected to air compressor by hose are also lowered along with 1-2 ropes as a backup for the hanging diver and provision of additional tools if needed. The silodiver sits on a special suspended on a rope chair and rotates a long-armed air jet around the silo wall. Of course he (no women are reported to exercise this job) is well dressed and equipped with a respiratory means.

In practice none of the silo owners agree making other holes or other changes in the silo building construction. This is one of the main constraint in the development of the robotic solution. From one side the inside dimension is several meters, and from another side the space for entering the silo is not bigger than about a meter. This means the cleaning robot construction must be foldable during transportation and before installation, and expandable after the robot is placed inside the silo. Clearly, this condition is very similar to the solar panels for the ISS (International Space Station) which have to be very compact for transportation and large enough when in the Space.

The SIRO, aimed to substitute a trained silo-cleaning worker in operating inside the confined space, must fulfill the following design requirements.

- Workplace requirement: SIRO should be transported to the silo roof taking into account that there is another roof over the silo's roof $(2-3 m$ height), therefore it should have appropriate weight and size, or it should be able to assemble its parts at the working place.

- Size: the robot's form should be compact/foldable before entering the silo through a small manhole, then expandable to reach the silo walls.

- Mobility: The robot must be able to move inside the silo to scan the entire height from top to bottom, letting the cleaning tools reach every point of the interior surface.
- Stabilization around any operational point in the interior 3D silo space; the robot should hold the position during the cleaning process when the cleaning tools rotate around the walls.

- Possibility to carry the respective cleaning tools and supporting equipment;

- Perception of the interior silo environment, more specifically its surface for quality assessment of the cleaning process.

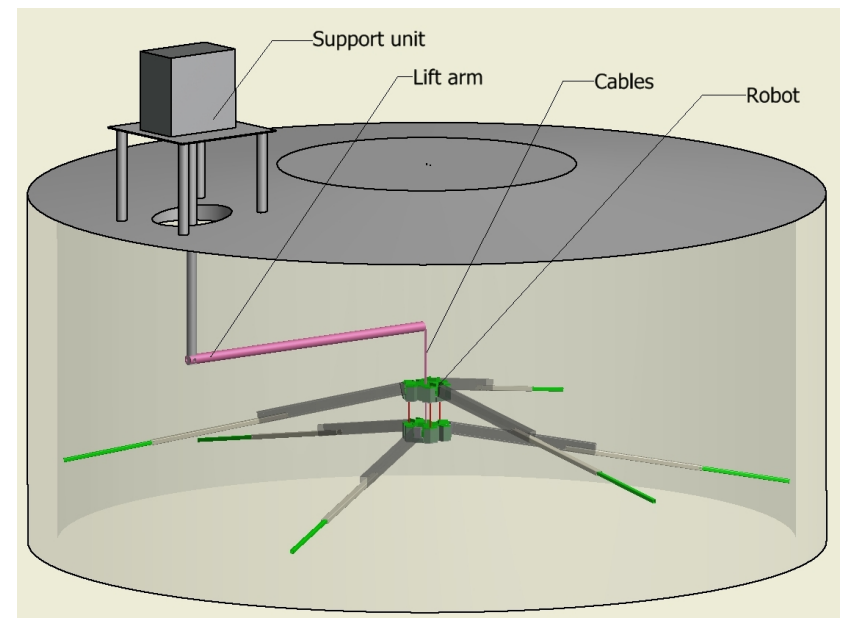

(a) The working pose

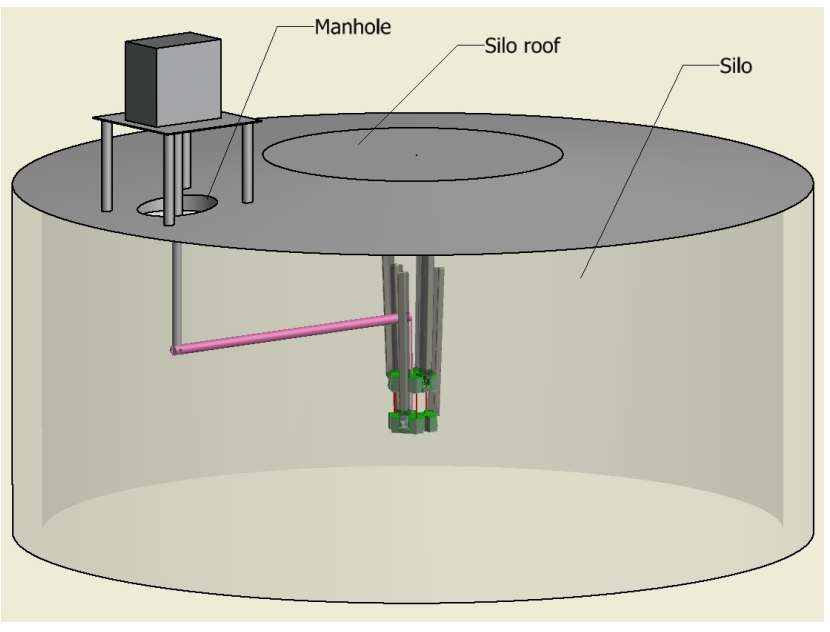

(b) The initial pose

Fig. 1. The proposed SIRO cleaning robot inside a silo

We propose the concept shown on Fig. 1 that appears to meet all these requirements. The entire silo cleaning system consists of three main parts:

- the cleaning robot with two platforms - in opened form on Fig. 1(a) and in retracted form on Fig. 1(b), and end effector mechanism;

- a support unit consisting of: control unit for all functions of the system; lift arm for positioning the cleaning robot at the silo central axis and spools of steel cables with respective driving motors used for robot suspension and motion; the steel cables coming from the support unit through the lift arm to the robot; 
- cleaning tools attached to the end effector (not shown on the figures) that rotate around the vertical robot axis, i.e. around the silo vertical axis.

The focus of this paper is at the cleaning robot and its basic motions inside the silo needed for surface cleaning. The support unit stays outside the confined space and provides supplementary functions to the robot. The set-up of the entire system is done by human operators before the robot enters the silo space. When cleaning is finalized, robot leaves the silo space using the support unit driven again manually by human operators. In the remaining part of this paper we do not discuss details of the support unit and the cleaning tools as they are not essential for the proposed concept.

\section{THE SIRO CONCEPT}

Moving the cleaning tools inside the silo and reaching every point of its interior surface is the substantial function for SIRO. A classical solution to achieve this functionality is to use two movements: one translational movement of the tools along the silo's vertical axis and rotation around that axis. The implementation of each movement is not a simple task itself due to the large dimension of the space. Vertical linear movement on a height of $20-30 \mathrm{~m}$ implies the use of suspension principle, where the gravity can be utilized appropriately. As any climbing robot cannot stay firmly on the silo wall due to extremely bad conditions for adhesion, the only feasible way is to suspend the robot bearing the cleaning tools equidistantly to the wall as shown on Fig. 1. The vertical robot position is changed by varying the length of the suspension steel cable.

Such central position of the robot is good in order to enable the second movement of the cleaning tools - rotational scanning of entire cylindrical surface. During this rotation, centrifugal forces and any small imbalance of the tools can create unpredicted (e.g. swinging) motions of the suspension steel cable that can cause physical interaction between the tools and the wall, even damage of the tools. Therefore, a stabilization of the center of rotation is strongly needed. It is achievable by using minimum three supporting arms which contact the wall, Fig. 1(a). In addition, these arms have to "climb" up/down for scanning the entire surface, which is accomplished by crawling type of movement.

The support unit installed over the inspection hole on the silo roof, Fig. 1(a), bears the entire cleaning robot. The two spools provide steel cables connected to the robot through the lift arm, called "suspension cable" and "crawling cable" respectively. The lengths of these cables are controllable correspondingly to the crawling type of motion explained later. In addition, they are also used for safety. The initial setup of the lift arm inside the silo is manual to bring the robot at the geometric center of the silo, where the suspension steel cable gives the robot's vertical position.

SIRO consists of three main parts: two identical platforms and end effector mechanism. The end effector holds the cleaning tools, and is attached under the bottom platform (not shown herein as it depends on the cleaning tools). The rotational speed of the tools depends on the cleaning process.

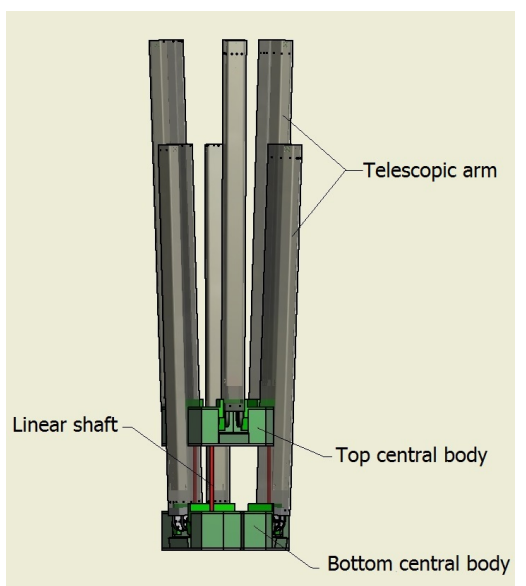

(a) The platforms and the arms in initial pose

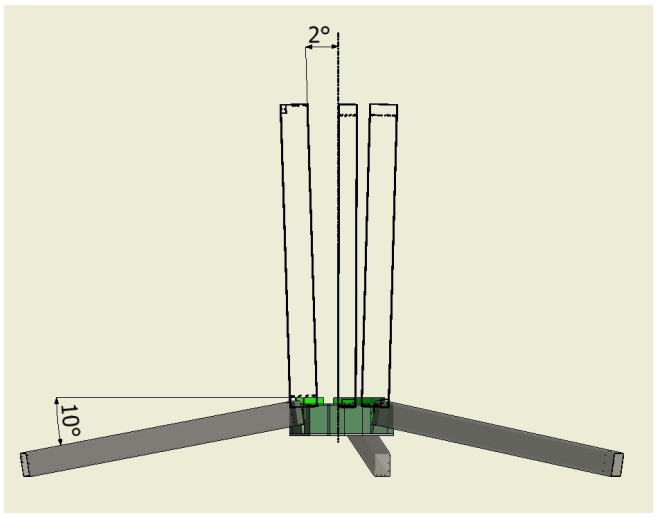

(b) The arms of one platform in opened pose (retracted)

Fig. 2. The two robot platforms and arms

Each platform consists of three telescopic arms and one central body as shown on Fig. 2 (zoomed-in). The central body has a hexagonal prism shape and the three arms are connected to its lateral surface by pivot joint with an angular shift of $120^{\circ}$ between each other. Three linear bearings are fixed in the central body to realize the linear movement between the two platforms by means of three free moving linear shafts. The two platforms are placed one over other with angular shift of $60^{\circ}$ around the vertical axis. The suspension cable is attached to the top platform and the crawling cable is attached to the bottom one. When suspension cable is fixed, one may pull/release the crawling cable for moving the bottom platform up/down.

The telescopic arm with $1500 \mathrm{~mm}$ length in retracted form consists of three segments (Fig. 3) of lengths $1500 \mathrm{~mm}, 1400 \mathrm{~mm}, 1300 \mathrm{~mm}$ can reach in extend form a length of $2400 \mathrm{~mm}$. The first segment of the telescopic arm contains the driving motor with ball screw mechanism that transfers the motion to next segments by a system of pulleys and ropes. When segment 2 starts to extend, cable 1 and pulley 1 force segment 3 to extend simultaneously with segment 2 . When segment 2 retracts, pulley 2 and cable 2 retracts segment 3 at the same time, so segment 3 always extends/retracts with segment 2 . 


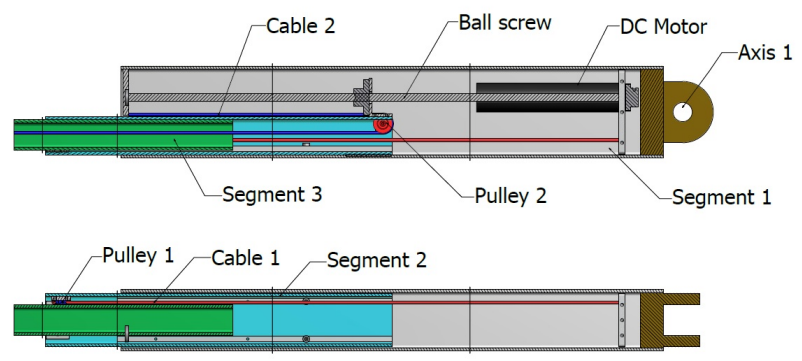

Fig. 3. Structure of the telescopic arms

\section{BASIC LOCOMOTION}

In this section we describe the main matters related to robot locomotion inside the silo. First, the preparation of the robot and its elements to serve is presented, then how to immobilize the robot during the time cleaning takes place is shown. Finally, the crawling vertical locomotion is outlined.

\section{A. Entering and placing inside the silo}

The robot is transported to the silo roof in folded configuration. The arms of the robot's two platforms are fully retracted and folded around their Axis 1 (Fig. 3) as shown on Fig. 2(a). In this configuration the robot size is sufficiently small to enter the manhole, Fig. 1(b). Then, after attached to the suspension and crawling cables, the robot is lowered into the silo by using these cables and the lift arm, which is lowered in the same time.

When the robot takes its highest position inside the silo, all arms of both platforms are released and they start to rotate about $100^{\circ}$ around Axis 1 ((Fig. 3) until reaching about $10^{\circ}$ angle with respect to the horizontal plane, Fig. 2(b), which is determined by a shoulder on the central bodies of platforms. The speed of releasing the arms is chosen to minimize any swinging or other undesired motions of the robot. The robot stays suspended by both cables.

\section{B. Holding a position}

To hold a vertical position, the arms of at least one of the platforms have to extend until they get contact to the interior silo surface. Due to the same extension speed, the arms should have the same extended lengths, therefore the robot will be almost centered in the cylindrical silo. The suspension cable neutralizes the gravity force $F_{\text {gravity }}$ caused by the robot's mass. When the arms reach the interior surface, the suspension cable is slightly released, therefore $F_{\text {gravity }}$ is no more compensated, hence it causes increased contact force. The friction force between the arms and the vertical surface must be bigger than the gravity force depending on the mass of the robot, $F_{\text {friction }}>F_{\text {gravity }}$, as shown on Fig. 4.

The appropriate friction force is generated by a pushing force $F_{\text {push }}$ produced by the ball screw mechanisms in the arms, and by increasing the friction coefficient between the arm and the wall. Furthermore the arch shape formed by the robot arms configuration reinforces the stability as well,

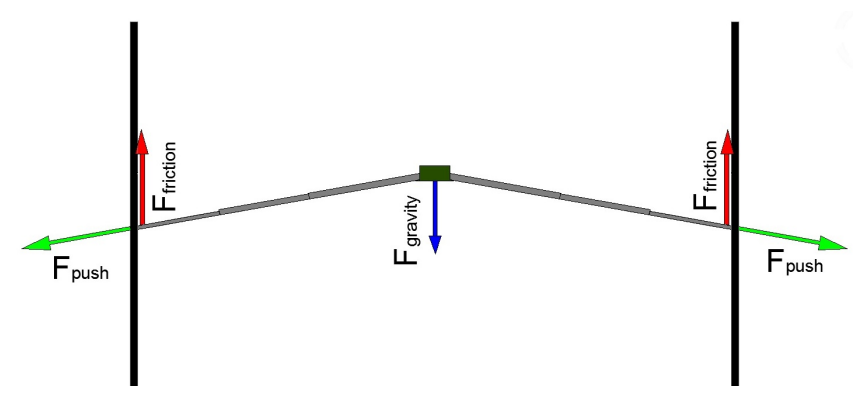

Fig. 4. Simplified forces diagram for one platform

where the central body of the platform plays the role of a keystone in the arch. At every moment at least one platform should be able to hold robot's weight and prevent sliding.

During the cleaning process the robot is not moving, i.e. the arms of both platforms are in contact to the silo wall, hence the stability of the robots is even more enhanced. When the robot is to move up/down, the arms of only one platform are in contact. If none of the arms is in contact, the robot platforms stay on the same height, as the suspension and crawling cables provide the support.

\section{Vertical movement}

The movement inside the silo space is achieved by a vertical crawling. The step of this crawling is determined by the distance between the two platforms, which depends on the cleaning area that the cleaning tools have to scan. The crawling is carried out by small sequential retracting/expanding of the platform arms followed by sequential pull/release of the suspension and crawling cables.

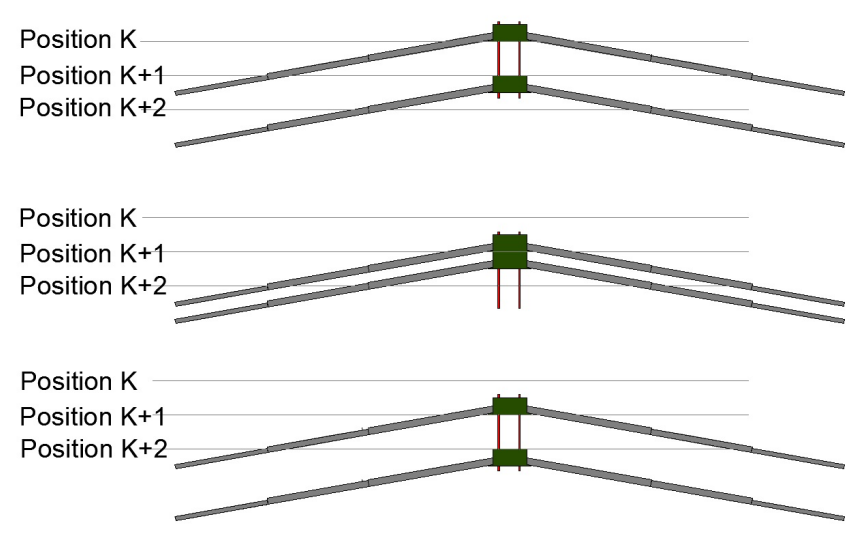

Fig. 5. Crawling movement inside silo space

Figure 5 illustrates the crawling. Three consecutive equidistant positions $\mathrm{K}, \mathrm{K}+1$ and $\mathrm{K}+2$ are to be used, the distance is equal to the constructive distance between both platforms. To make a step down, the two platforms should follow the following sequence of actions:

- The initial state is: top platform at position $\mathrm{K}$, bottom platform at position $\mathrm{K}+1$.

- After one step down crawling is initiated, the bottom platform stays at $\mathrm{K}+1$ (crawling cable doesn't change). 
- Suspension cable is on hold, top platform arms retract at about $1 \%$ of their length to release contact to wall.

- Suspension cable is released and top platform by its own weight moves down slowly along the linear shafts.

- When top platform reaches position $\mathrm{K}+1$, suspension cable in on hold and top platform arms expand until getting contact to wall - top platform is at its new position.

- Bottom platform arms retract at about $1 \%$ of their length to release contact to wall.

- Crawling cable is released and bottom platform by its own weight moves down slowly along the linear shafts.

- When bottom platform reaches position $\mathrm{K}+2$, crawling cable in on hold and bottom platform arms expand until getting contact to wall - bottom platform is at its new position.

Similar sequence of actions can be used for making a step up, however in this case the suspension and crawling cables have to be pulled up, instead of released. It should be noted that normally the cleaning process is executed from the top to the bottom of the silo. All particles and remaining material from the wall will go down by gravity and leave the upper part of the silo already cleaned. Therefore, when the silo bottom is reached, the cleaning is considered completed and both cables are pulled up to bring the robot close to the silo top. After that a dedicated procedure of taking the robot out of the silo space is performed by humans.

\section{CONCLUSION}

A new concept of suspended robot for cleaning of interior silo surfaces is presented in this paper. The design is based on minimalistic approach to achieve a compromise between maximum performance and reasonable complexity and cost of the system. The system description here is limited only to the kinematic structure and main functionalities of the robot, as it is still in a process of prototyping.
The robot has a foldable and expandable structure in order to meet two very contradictory conditions, namely very large space to operate in and very small entry hole on the silo roof. Actually, the robot main functions are to bear the cleaning tools (air jets) and to stabilize the cleaning tools while they rotate performing the cleaning. The crawling vertical movement of the robot and the rotation of the cleaning tools permit reaching the entire interior surface.

The robot is characterized by using minimum degrees of freedom and gravity is utilized in best way for reducing the power needed for locomotion. Respectively, the control system is simplified, as the control action sequence has minimal complexity.

\section{REFERENCES}

[1] Confined Spaces Guideline, Occupational Health and Safety Branch Ministry of Labour, 2009.

[2] Airmatic Inc. Silo cleaning. [Online]. Available: http://www.airmatic.com/service-group-silo-cleaning.html

[3] Primasonics. Acoustic cleaner. [Online]. Available: http://www.primasonics.com

[4] M. Ryffel, "Device and methode for the treatment for a container wall and container," Jun 2009, uSA patent, US 2009/0144917 A1.

[5] Silo-RoBoFox . [Online]. Available: http://www.Silo-RoBoFox.de

[6] S. W. Glass and F. C. Klahn, "ReTRIEVR, A long-reah robot for tank or silo waste retrieval ," in Proc. of Waste Managment 2001 Conference, February 25 - March 12001.

[7] A. Goldenberg, M. Gryniewski, and T. Campbell, "Aarm: A robot arm for internal operations in nuclear reactors," in Proc. 1st Int Applied Robotics for the Power Industry (CARPI) Conf, 2010, pp. 1-5.

[8] H. J. Lee, J. K. Lee, B. S. Park, K. Kim, and H. D. Kim, "Development of an overhead crane for remote handling tasks at nuclear facility," in Proc. Int Control Automation and Systems (ICCAS) Conf, 2010, pp 1830-1834.

[9] D. Lee, N. Ku, T.-W. Kim, K.-Y. Lee, J. Kim, and S. Kim, "Selftraveling robotic system for autonomous abrasive blast cleaning in double-hulled structures of ships," Automation in Construction, vol. 19, no. 8, pp. 1076-1086, Dec 2010.

[10] X. Gao, Z. Jiang, J. Gao, D. Xu, Y. Wang, and H. Pan, "Boiler maintenance robot with multi-operational schema," in Proc. IEEE Int. Conf. Mechatronics and Automation ICMA 2008, 2008, pp. 610-615.

[11] H. Zhang, J. Zhang, R. Liu, W. Wang, and G. Zong, "Design of a climbing robot for cleaning spherical surfaces," in Proc. Robotics and Biomimetics (ROBIO). 2005 IEEE Int. Conf, 2005, pp. 375-380. 